\title{
Symposium der C. E. Alken-Preisträger in Heidelberg Dezember 1992
}

\author{
K.-P. Jünemann \\ Urologische Klinik (Direktor: Prof Dr. P. Alken), Fakultät für Klinische Medizin Mannheim der Universität Heidelberg
}

\section{E. Alken-Preis 1993}

$J$. W. Thüroff, Wuppertal: Die Faszienzügelplastik zur Behandlung der Streßinkontinenz des Mannes. Koautoren der Arbeit waren: $M$. Hohenfellner, D. Schultz-Lampel

Die Harninkontinenz des Mannes stellt immer noch ein therapeutisches Problem dar. Physikalische und konservativ-medikamentöse Behandlungen bieten eine limitierte Rehabilitationsbreite, so daß in der Mehrzahl der Fälle eine rekonstruktive Operation erforderlich wird. Die große Zahl unterschiedlicher Operationsmethoden zur Therapie der Harninkontinenz bei Mann und Frau lassen sich in vier unterschiedliche Verfahren klassifizieren: 1. Rekonstruktion des quergestreiften Sphinktermechanismus, 2. funktionelle Rekonstruktion des glattmuskulären Sphinktermechanismus, 3. Urethrakompression und 4. Urethraersatz. Ungeachtet der Vielzahl der angewandten Verfahren sind die operativen Ergebnisse zur Behandlung der Streßinkontinenz des Mannes nach wie vor unbefriedigend. Schlingenoperationen mit nichtdehnbaren Materialien (Faszie, Faszie mit Muskel, Kunststoffband etc.) fanden auch in der Behandlung der männlichen Harninkontinenz Verwendung. Ungeachtet einer Erfolgsrate von $89 \%$ exzellenten und 10\% guten Ergebnissen bei 133 in der Literatur berichteten Fällen, hat sich diese Operationstechnik bei der männlichen Harninkontinenzbehandlung nicht durchsetzen können, nicht zuletzt aufgrund fehlenden Grundlagenwissens über die Funktion der Schlingentechniken. Gegenwärtig stellt die Implantation einer steuerbaren, alloplastischen, hydraulischen Sphinkterprothese die am weitesten verbreitete Operationstechnik dar.

Unter diesem Aspekt wurden von Thüroff et al. tierexperimentelle Untersuchungen zum aktiven Mechanismus der reflektorischen Kontraktion der quergestreiften Sphinktermuskulatur durchgeführt sowie Studien über den Einfluß der Faszienzügelplastik auf den urethralen Verschlußmechanismus unter Streßbedingungen. Dabei konnte gezeigt werden, daß durch eine Faszienzügelplastik der urethrale Verschlußmechanismus unter Streßbedingungen erheblich zu verbessern ist, woraufhin der Entschluß gefaßt wurde, durch Sektionsstudien an der Leiche eine modifizierte Technik der Faszienzügelplastik zur Therapie der Streßinkontinenz des Mannes zu erarbeiten.

Akt. Urol. 24 (1993) 304-314

(c) Georg Thieme Verlag Stuttgart · New York

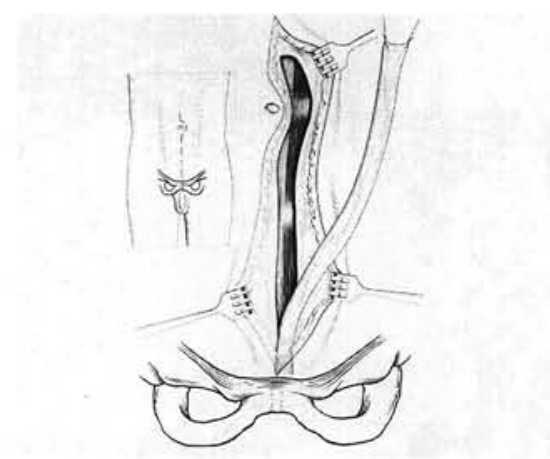

Abb. 1 Bildung der Faszienschlinge aus der Rektusscheide.

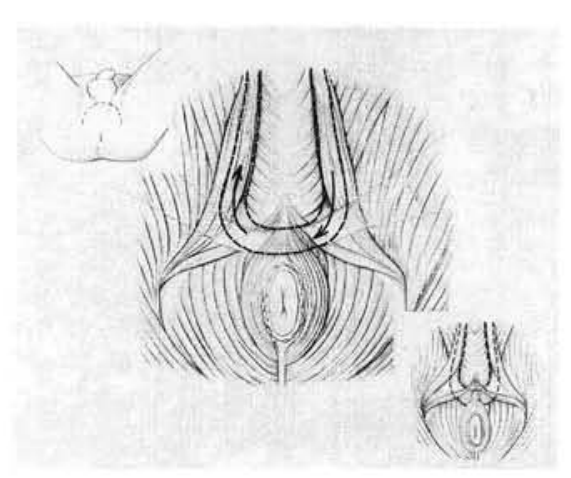

Abb. 2 Darstellung der Perinealregion.

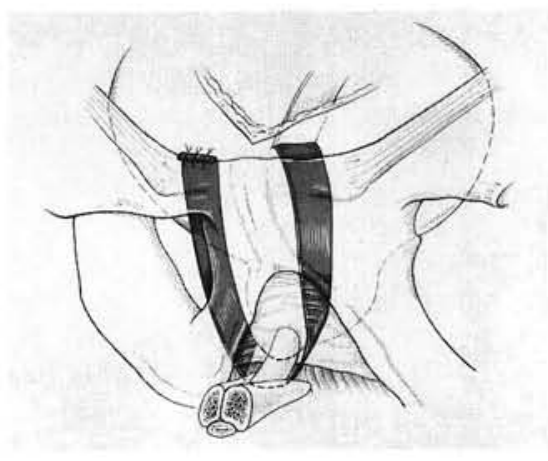

Abb. 3 Fixation der durchgeführten Faszienschlinge am Cooperschen Ligament.

Die daraus entstandene Modifikation wurde seit 1988 bei 22 Männern im Alter von 29 bis 81 Jahren mit zystomanometrisch nachgewiesener Streßinkontinenz durchgeführt. In erster Linie handelt es sich um Patienten mit Streßinkontinenz nach transurethraler Prostataresektion, suprapubischer Adenomektomie oder radikaler Prostatektomie. 
Die operative Technik stellt sich wie folgt dar: Zur Bildung der Faszienschlinge wird ein 1,5 bis $2 \mathrm{~cm}$ breiter und etwa $20 \mathrm{~cm}$ langer, an der Symphyse gestielter Faszienstreifen aus der vorderen Rektusscheide exzidiert (Abb. 1). Nach Eröffnung des Cavum Retzii wird die endopelvine Faszie dargestellt und beidseits inzidiert, die als Ein- und Austrittspforte für den Durchzug des präparierten Faszienstreifens dient. In Steinschnittlage wird über einen umgekehrten, hufeisenförmigen Schnitt um den Anus das Perineum dargestellt, ohne das Centrum tendineum zu durchtrennen (Abb. 2). Die fertige Faszienschlinge wird durch die Faszieninzisionen durchgeführt, umschlingt teilweise den M. pubococcygeus und kommt kranial des Diaphragma urogenitale (Mm. transversum perinei superficialis et profundus) zu liegen. Das freie Ende des Faszienstreifens wird mit einer eingeführten Klemme in die Fossa ischiorectalis eingezogen und nach Umschlingung der Prostata bzw. membranösen Harnröhre durch die rechte Fossa ischiorectalis, durch den präparierten Tunnel, nach abdominal durchgezogen, so daß sich eine Faszienschlinge ergibt, die unter kräftiger Spannung mit 3/0 Prolenenähten am Cooperschen Ligament fixiert wird (Abb. 3).

Bei einer durchschnittlichen Nachbeobachtungszeit von 10,3 Monaten waren 14 der 22 operierten Patienten vollständig kontinent, bei 2 Patienten hat sich die Sympomatik deutlich gebessert. 6 Patienten wurden als weiterhin inkontinent klassifiziert. 5 dieser $6 \mathrm{~Pa}$ tienten hatten initial eine totale Inkontinenz (in 4 Fällen nach radikaler Prostatektomie). Alle Patienten können spontan miktionieren.

Aufgrund der guten Resultate $(72,7 \%)$ kommen Thüroff et al. zu dem Schluß, daß die klinischen Ergebnisse der modizifierten Faszienzügelplastik zur operativen Korrektur der Harninkontinenz des Mannes eine erfolgreiche Behandlungsmethode darstellt.

\section{Operative Urologie}

G. E. Voges, Mainz: Nervenschonende und nicht-schonende radikale retropubische Prostatektomie: Inzidenz und klinische Signifikanz tumorpositiver Resektionsränder. Koautor dieser Arbeit war R. Hohenfellner

Die Entwicklung von Operationstechniken mit geringer Morbidität und mit Erhalt von Lebensqualität (Potenzerhaltung) ist einer der wesentlichen Gründe für die herausragende Rolle, die die radikale Prostatektomie heute in der Behandlung des klinisch organbegrenzten Prostatakarzinoms spielt. Die nervenerhaltenden Operationstechniken - mit Dissektion unmittelbar an der Prostataoberfläche - werfen allerdings die Frage der möglichen Beeinträchtigung der Radikalität des Eingriffs auf ('cancer sparing surgery').

Anhand einer kompletten, computergestützten Aufarbeitung und Rekonstruktion von 241 konsekutiven Prostatektomiepräparaten aus der Stanford-Serie (Gruppe I) und 100 konsekutiven aus der Mainzer Klinik (Gruppe II) wurden tumorpositive Präparatränder exakt lokalisiert und ihre Beziehung zu einer Kapselpenetration des Karzinoms bzw. operationstechnischen Fehlern (Dissektion innerhalb der natürlichen Grenzen der Prostata) geklärt. Ein besonderes Augenmerk galt dem Einfluß der angewandten Operationstechnik auf Frequenz und Art (assoziiert mit Kapseldurchbruch des Karzinoms vs. chirurgischer Fehler) der positiven Absetzungsränder. Bei 117 der 241 Patienten in Stanford war eine unilaterale nervenerhaltende Operation kontralateral zum palpablen Tumor erfolgt, bei 31 mit nicht-palpablen Karzinomen (Stadien T1a-c) eine bilaterale nervenerhaltende Dissektion. Bei 93 der 241 Patienten in Stanford und allen 100 in Mainz wurde auf den Nervenerhalt verzichtet. In Stanford wurde entsprechend einer Modifikation der Walsh-Technik aszendierend, in Mainz deszendierend operiert.

68 der 241 Präparate $(28,2 \%)$ der Stanford-Serie hatten tumorpositive Ränder und 31 von 100 (31\%) in der Mainz-Serie (Tab. 1). 41,2\% (28/68) der positiven Ränder in Gruppe I waren durch einen chirurgischen Fehler (iatrogen) verursacht, in Gruppe II lag in 7 von 31 Fällen (22,6\%) ein chirurgischer Fehler vor. Mit jeweils $45 \%$ war der Prostataapex die häufigste Lokalisation tumorpositiver Absetzungsränder in beiden Gruppen. Chirurgische Fehler in Gruppe I kamen am häufigsten dorsolateral im Bereich des neurovaskulären Bündels vor, während in Gruppe II die rektale Oberfläche der Prostata bevorzugt betroffen war.

Die Frequenz iatrogen bedingter tumorpositiver Absetzungsränder nahm in Gruppe I von 5,4\% (5/93), bei Fällen ohne nervenerhaltende Technik, auf $14,5 \%(17 / 11 /)$ mit unilateralem Nervenerhalt zu. Bei bilateralem Nervenerhalt fand sich ein weiterer Anstieg auf $19,4 \%(6 / 31) .16$ von $23(69,6 \%)$ der iatrogen bedingten positiven Absetzungsränder bei nervenerhaltender Operationstechnik fanden sich auf der Seite des Nervenerhalts und 12 dieser 23 Karzinome waren mit Ausnahme der positiven Resektionsränder komplett auf die Prostata be- tumorpositive Präparatränder

\begin{tabular}{lrlrrrr} 
& \multicolumn{7}{c}{ tumorpositive Präparatränder } \\
& $n$ & Gesamt & $\%$ & $\mathrm{KP}+$ & $\mathrm{KP}-$ & $\mathrm{KP}+/ \mathrm{KP}-$ \\
\hline Gruppe I (Stanford) & & & & & \\
Gesamt & 241 & 68 & $(28,2 \%)$ & 40 & 28 & - \\
nicht nervenerhaltend & 93 & 30 & $(32,3 \%)$ & 25 & 5 & - \\
unilat. nervenerhaltend & 117 & 28 & $(23,9 \%)$ & 11 & 17 & - \\
bilat. nervenerhaltend & 31 & 10 & $(32,3 \%)$ & 4 & 6 & - \\
Gruppe II (Mainz) & & & & & & 3 \\
nicht nervenerhaltend & 100 & 31 & $(31,0 \%)$ & 24 & 4 & 3 \\
\hline
\end{tabular}

Tab. 1 Tumorpositive Resektionsränder in Beziehung zur angewandten Operationstechnik

$(\mathrm{KP}+=$ assoziiert mit einer Kapselpenetration des Tumors, $\mathrm{KP}-=$ chirurgischer Fehler) 
grenzt. Das Risiko, einen tumorpositiven Absetzungsrand auf der nervenerhaltend operierten Seite durch einen chirurgischen Fehler $\mathrm{zu}$ induzieren, lag insgesamt bei $10,8 \%(16 / 148)$. In $8,1 \%$ der Fälle (12/148) betraf dies Präparate mit ansonsten komplett organbegrenzten Tumoren.

Tumorrezidive (PSA $>0,3 \mathrm{ng} / \mathrm{ml}$, Yang, Nachsorge $>18$ Monate) traten bei komplett organbegrenzten Tumoren in Gruppe I in 6,7\% der Fälle auf. Bei organüberschreitendem Tumorwachstum fanden sich Rezidive in 36,8\%. Lagen positive Absetzungsränder vor, betrug die Rezidivquote 54,4\%. Besonders interessant war der Vergleich zwischen den komplett organbegrenzten Karzinomen und den komplett begrenzten Tumoren mit positiven Präparaträndern. Während in erstgenannter Gruppe die Rezidivrate bei $6,7 \%$ lag, betrug sie in letztgenannter $29,4 \%$.

Schlußfolgerung: Tumorpositive Absetzungsränder bei der radikalen Prostatektomie sind einerseits durch Tumor bedingt, der die natürlichen Grenzen der Prostata überschreitet und andererseits durch chirurgische Fehler bei zu naher Dissektion an der Prostataoberfläche. Chirurgische Fehler werden bevorzugt bei der nervenerhaltenden Operationstechnik gefunden, aber auch bei anderen Techniken besteht das Risiko des iatrogen bedingten positiven Resektionsrandes. Im Sinne der Tumorchirurgie ist die Indikation zur nervenerhaltenden Technik extrem zu limitieren. Auch bei nicht nervenerhaltender Operation ist auf die sorgfältige Dissektion außerhalb der natürlichen Grenzen der Prostata zu achten.

A. Knipper, Lübeck: Endoskopische Techniken zur Inzision von Harnleiterstrikturen. Koautoren dieser Arbeit waren: C. Durek, D. Jocham

Harnleiterstrikturen werden unterteilt in kongenitale Stenosen (Ureterabgangsstenose, Harnleiterklappen, obstruktiver Megaureter) und erworbene Stenosen im Gefolge offener operativer Eingriffe, endourologischer Manipulation oder nicht-operativer Ursachen (Radiatio, Entzündungen, externe Ureterkompression etc.). Dilatation des oberen Harntrakts mit nachfolgend rezidivierenden Harnwegsinfektionen sind die Folge, die bei parenchymatöser Mitbeteiligung die renale Funktion beeinträchtigen und im vollständigen Nierenfunktionsverlust enden können. In Abhängigkeit von der Strikturlokalisation wurden unterschiedliche chirurgische Techniken zur Strikturbeseitigung entwickelt, z. B. Psoas-Hitch-Technik, Ureteropyeloplastik, Autotransplantation. Endoskopische Therapieverfahren von Harnleiterstrikturen beinhalten die konservativen Behandlungsformen der inneren Harnleiterschienung oder die Ballondilatation, ebenso wie die endoskopischen Schnittechniken, basierend auf der Idee von Davis (1943), der intubierten Ureterotomie mittels Messer- oder Laserinzision. Gemäß Literatur beträgt die Erfolgsrate der Nicht-Laserinzisionsverfahren zwischen 50 und $90 \%$. Die Verwendung von Laserinzisionssystemen liegt in der Vorstellung begründet eine Ergebnisverbesserung durch geringere Invasivität und Morbidität zu erreichen, nicht zuletzt durch Miniaturisierung und der damit verbundenen Flexibiliät von sehr kleinen Faserdurchmessern sowie einer weniger traumatisierenden, definierten Energie.

Da keine adäquaten Tiermodelle zur experimentellen Untersuchung der Behandlung von Ureterstrikturen existieren, wurden unterschiedliche Inzisionsverfahren in In-vitro-Experimenten erprobt und anschlieBend ein experimentelles Tiermodell entwickelt, wobei die Laserinzision mit der Elektro- bzw. der Messerinzision verglichen wurde. Dazu wurden 540 frische Schweineureteren mit dem XT-Holmium:YAG-Laser inzidiert, um die besten Schnittparameter (Faserdurchmesser: 100$600 \mu \mathrm{m}$; Schnittgeschwindigkeit: $0,25-1 \mathrm{~mm} / \mathrm{s}$; Energiebreite: 1-6 W; 550-750 $\mu$ s kontinuierlicher Modus oder 40-120 $\mu$ s Pulsmodus) bestimmen zu können.

Jeder Harnleiterschnitt wurde fotografisch dokumentiert und anschließend in $5 \%$ igem Formaldehyd fixiert.

Anhand der anschließenden histologischen Aufarbeitung des inzidierten Ureterabschnittes mittels HE-Färbung wurden Defekttiefe, Koagulationstiefe sowie Defektausdehnung und Koagulationsausdehnung mit einem rechnergestützten Morphometriesystem bestimmt.

Die Ergebnisse zeigten, daß mit zunehmender Energie größere Gewebedefekte auftraten, eine Zunahme in der Defektausdehnung, die ca. $100 \mu \mathrm{m}$ größer war als der verwendete Faserdurchmesser, wohingegen die Koagulationstiefe mit $0,4 \mathrm{~mm}$ nahezu konstant blieb. Die Schnittgeschwindigkeit hatte keinen Einfluß auf histomorphologische Veränderungen. Tiefere Schnitte mit geringerer Energie in vergleichbarer atraumatischer Qualität wurden durch Wahl des Pulsmodus im Vergleich zu einem kontinuierlichen (running mode) Laserstrahl erreicht.

Aufgrund der erstellten Ergebnisse kamen Knipper et al. zu dem Schluß, daß Laserenergien zwischen 3 und $5 \mathrm{~W}$ im kontinuierlichen Modus oder 2-3W im Pulsmodus ausreichten, um die Ureterwand mit einem Schnitt $\mathrm{zu}$ inzidieren. Wegen Faserankoppelungsproblemen bei Faserdurchmessern von $100 \mu \mathrm{m}$ empfehlen die Autoren - aufgrund der In-vivo-Experimente - die Verwendung von $200-300 \mu \mathrm{m}$ Fasern.

Die Autoren kommen zu dem Schluß, daß nicht nur die Methode der Harnleiterinzisionstechniken, sondern ebenso die weiteren Schritte wie Harnleiterschienung, Durchmesser und Dauer der Ableitung weiteren Untersuchungen vorbehalten bleiben müssen.

M. Fisch, Mainz: Die Rekonstruktion der Ileozökalklappe. Koautoren der Arbeit waren: F. Spieß, R. Wammack, R. Hohenfellner

Rezidiveingriffe nach kontinenter Harnableitung, ebenso wie primäre Harnableitungstechniken nach vorangegangenen Darmoperationen, stellen den Operateur immer wieder vor das Problem des postoperativen Kurzdarmsyndroms. Unklar bleibt auch, welchen 
Einfluß die Resektion der Ileozökalklappe im Rahmen einer Mainz-Pouch-I-Anlage auf die zuvor beschriebene Pathologie nimmt und wie die physiologische Funktion dieses Sphinktermechanismus zu verstehen ist.

Tierexperimentelle Untersuchungen am Hund hatten gezeigt, daß die Ileozökalklappe nicht nur eine Sphinkterfunktion erfüllt, sondern die Transitzeit im Darm verlängert. Zusätzlich verhindert die Klappe einen koloilealen Reflux, so daß eine bakterielle Kolonisation des terminalen Ileums verhindert wird. Inwieweit dieses bakterielle Wachstum bei fehlender Ileozökalklappe die Resorptionseigenschaften des Ileums beeinträchtigen, bleibt dagegen unklar.

Bisher waren unterschiedlichste Operationstechniken ausprobiert worden, um die gastrointestinale Transitzeit zu verlängern (Inkorporation eines umgekehrten Darmsegments, ileo-ileale Intussuszeption, Rekonstruktion der Ileozökalklappe). Die Mehrzahl dieser Verfahren wurde nur experimentell an Hunden untersucht, in nur sehr wenigen Fällen wurden diese Verfahren am Menschen ausprobiert, ohne daß eine dieser Techniken eine weite Akzeptanz fand.

1984 beschrieb Vinograd eine OP-Technik, bei der das Konzept der submukösen Tunnelung bei Ureterneueinpflanzung für die Bildung eines artifiziellen Ventils im Gastrointestinaltrakt verwendet wurde. Die auf diese Weise operierten 25 Hunde mit rekonstruierter Ileozökalklappe zeigten keinerlei Obstruktion, auf der anderen Seite konnte eine vierfache Verzögerung der Transitzeit im Darm nachgewiesen werden, ebenso wie bei Verwendung einer Tunnellänge von $4 \mathrm{~cm}$ ein zökoilealer Reflux vermieden werden konnte.

Die eigenen Erfahrungen mit zwei unterschiedlichen Sphinktermechanismusformen bei kontinenter Harnableitung führten dazu, daß die Methode der Rekonstruktion der lleozökalklappe sowohl beim invaginierten Ileumnippel als auch beim submukös eingebetteten Appendix in die Taenia libera des Zökums untersucht wurde. Bei 15 Hunden wurden $7 \mathrm{~cm}$ des Colon ascendens und 2 Ileumschlingen der gleichen Länge reseziert. Bei 5 Hunden wurde eine normale End-zu-End-Anastomose durchgeführt, wohingegen in den anderen 10 Fällen die Ileozökalklappe rekonstruiert wurde. Das terminale Ileum wurde invaginiert zur Bildung eines Nippels von $4 \mathrm{~cm}$ Länge. Der mittels 2 Staplerreihen bei 3 und $9 \mathrm{Uhr}$ gesicherte Nippel wurde in das Colon ascendens eingezogen und die Grenzflächen zwischen lleum und Kolon mittels 4/0 Einzelknopfnähten anastomosiert.

Bei der zweiten Technik wurde das Colon longitudinal inzidiert und die muskuläre Schicht von der Mukosa abpräpariert, so daß sich ein breites Bett für die submuköse Einbettung des Ileums bildete (Abb. 1). Am kranialen Ende wurde die Mukosa inzidiert und die lleumschlinge mit den Grenzflächen dieser Inzision anastomosiert (Abb. 2). Anschließend wurde die seromuskuläre Schicht über dieser Anastomose geschlossen, so da $ß$ sich auch hier ein $4 \mathrm{~cm}$ langer submuköser Tunnel bildete (Abb. 3, 4, 5). Der Nachbeobachtungszeitraum betrug 8 Monate, bis zu diesem Zeitpunkt war in jeder Gruppe ein

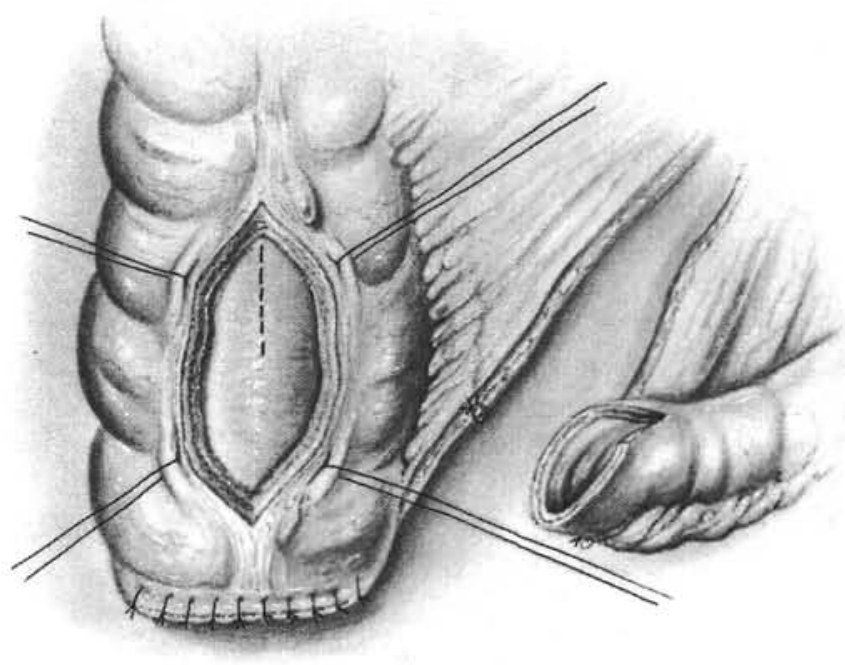

Abb. 1 Längsinzidiertes Kolon mit darunter erhaltener Mukosa.

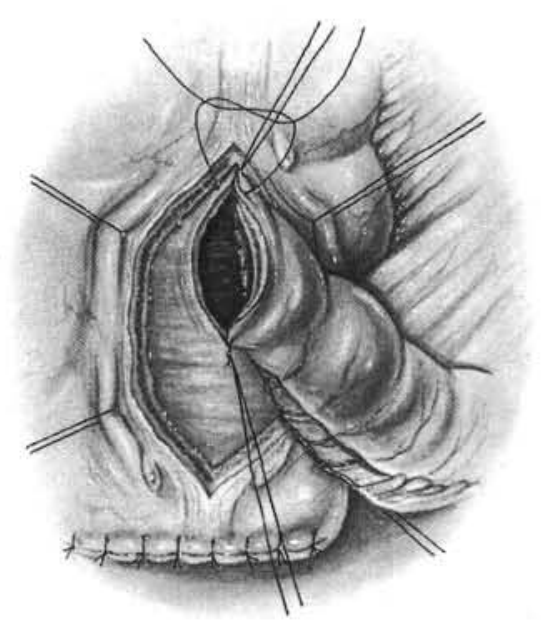

Abb. 2 Kraniale Mukosaeröffnung mit koloilealer Anastomose.

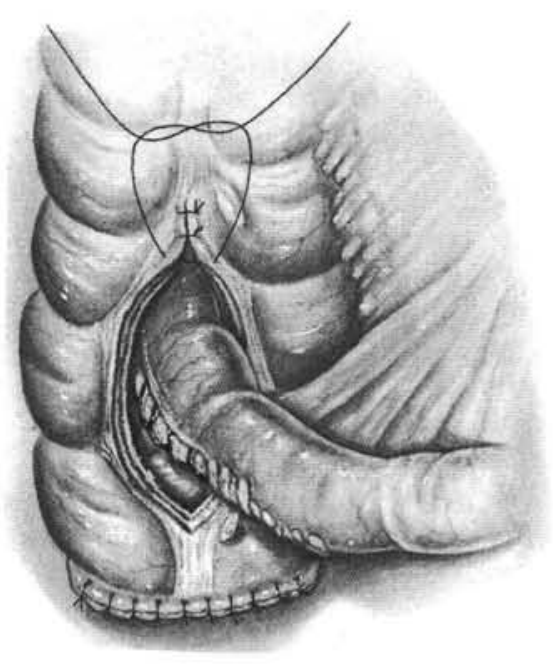

Abb. 3 Seromuskuläre Naht mit Bildung eines $4 \mathrm{~cm}$ langen submukösen Tunnels. 


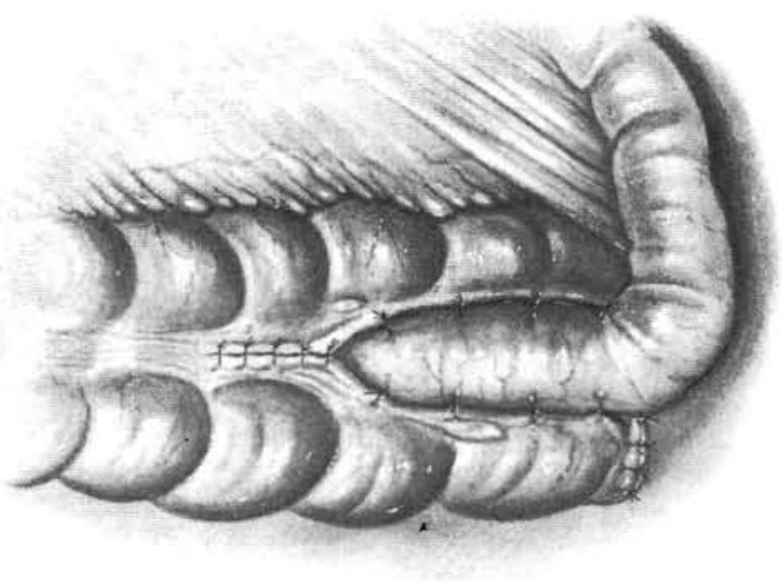

Abb. 4 Fertiggestellter submuköser Tunnel.

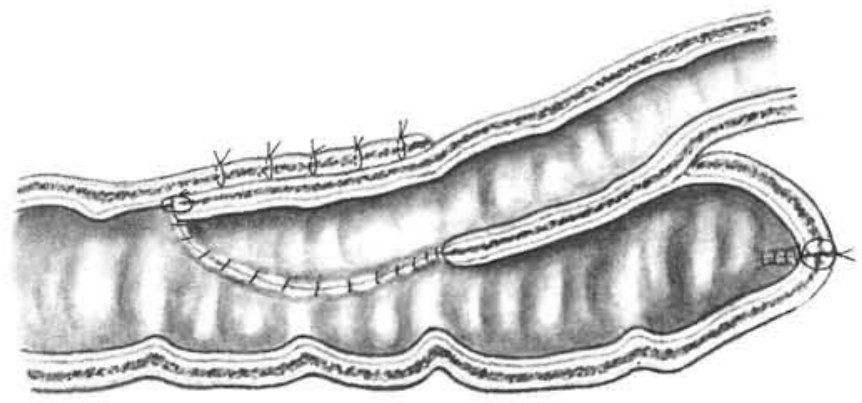

Abb. 5 Rekonstruierte lleozökalklappe von seitlich.

Hund verstorben, wobei dies nicht auf die Operation zurückgeführt werden konnte.

Die berichteten Langzeitergebnisse zeigen, daß der Verzicht auf Stapler sowie die Rekonstruktion der ileozökalen Klappe als submukös eingebettete Dünndarmschlinge dem intussuszeptierten Nippel deutlich überlegen ist, woraufhin die Mainzer Gruppe diese Technik bei bisher 6 Patienten eingesetzt hat. In allen Fällen lag eine ausgedehnte präoperative Diarrhö, aufgrund von Radiatio oder Darmresektion, vor. Der postoperative Verlauf war bei allen Patienten komplikationslos, in keinem Fall trat eine Ileussymptomatik ein. Die präoperative Stuhlfrequenz war postoperativ gleich oder verbesserte sich, so daß Fisch et al. zu dem Schluß kommen, daß Patienten mit ausgedehnter Darmresektion, Radiatio oder bereits präoperativ vorliegender Kurzdarmsymptomatik eine Rekonstruktion der Ileozökalklappe erhalten sollten, die im Rahmen der kontinenten Harnableitung problemlos durchgeführt werden kann.

T. Esen, Mannheim (Istanbul): In-vitro-Modell zur Evaluierung hydrodynamischer Aspekte unterschiedlicher Ventilmechanismen bei der kontinenten Harnableitung. Koautoren dieser Arbeit waren: K.-P. Jünemann, P. Alken

Die sehr attraktive Möglichkeit eine afunktionelle oder tumorbefallene Harnblase durch eine Darm- blase zu ersetzen, hat in den vergangenen 10 Jahren zunehmend an Popularität gewonnen. Gegenwärtig stehen beinahe 20 Techniken zur kontinenten Harnableitung oder totalem Blasenersatz mit detubularisierten Darmsegmenten zur Verfügung. Bei allen diesen Techniken ist und bleibt die Gewährung und Erhaltung der Harnkontinenz durch kompetente Ventilmechanismen eine echte Herausforderung.

Wie die Langzeitergebnisse verschiedener Methoden zur kontinenten Harnableitung zeigen, mußten alle Ventilmechanismen im Laufe der klinischen Studien zwei bis vier Modifikationen erfahren, ehe die initial sehr hohen postoperativen Komplikationen verbessert werden konnten. Selbst die Dünndarmintususzeption, die mit Abstand der populärste Kontinenzmechanismus ist und über ein von zahlreichen tierexperimentellen Studien abgeleitetes Grundlagenwissen verfügt, mußte einen langen klinischen Erfahrungsweg von mehr als 10 Jahren mit mehr als 600 Patienten zurücklegen, um die für die ersten 250 Fälle immerhin $62 \%$ Komplikationsrate bei den letzten 125 Patienten auf $13 \%$ zu reduzieren (Skinner, 1992). Nicht zuletzt die begrenzte Übertragbarkeit der tierexperimentellen Ergebnisse für den klinischen Einsatz waren verantwortlich für diese Entwicklung. Der aktuelle Stand fehlenden Grundlagenwissens über funktionelle Aspekte unterschiedlicher Ventilmechanismen war der Anlaß an einem In-vitro-Modell, das sowohl die Evaluierung der hydrodynamischen Eigenschaften dieser Kontinenzmechanismen als auch die Entwicklung neuer und alternativer Techniken ermöglichen sollte, zu untersuchen. An vom Schlachthof bezogenem frischen Schweinedarm wurden drei unterschiedliche Kontinenzmechanismen mit insgesamt vier Ventilarten untersucht: 1 . Nippelventile (2, 3, 4 und $5 \mathrm{~cm}$ ), a) Dünndarm, b) Dickdarm; 2. eingeengter Dünndarm in einer zökalen Tänie, a) Durchzug (3, 4, 5 und $6 \mathrm{~cm})$, b) Einbettung $(3 \mathrm{~cm}) ; 3$. terminales Ileum mit Reflux über Ileozökalklappe, a) Plikatur, b) Einengung. Zusätzlich wurde eine neue Kontinenztechnik entwickelt (Kolon-Serosa-Tunnel), indem ein $10 \mathrm{~cm}$ langer $12 \mathrm{Ch}$ Serosa-Tunnel auf der antimesenterialen Seite eines $15 \mathrm{~cm}$ langen Dickdarmsegments gebildet und bei 3, 5, 7 und $9 \mathrm{~cm}$ gefenstert wurde (Abb. 1). Über einen $12 \mathrm{Ch} 2$ Kanal-Zystometriekatheter wurden unter konstanter Flußrate von $30 \mathrm{ml} / \mathrm{min}$ Leckpunkt- und Maximaldruckwerte ermittelt. Der Durchschnittswert von jeweils 3 Messungen wurden berechnet. Die hydrodynamischen Messungen ergaben folgende Resultate: 1. Der Leckpunktdruck für die $2 \mathrm{~cm}$ Dünndarmintussuszeption lag bei $40 \mathrm{~cm} \mathrm{H}_{2} \mathrm{O}$. Die 3,4 und $5 \mathrm{~cm}$ langen Ventile zeigten maximale Druckwerte von 128, 115 und $145 \mathrm{~cm} \mathrm{H}_{2} \mathrm{O}$. Das $2 \mathrm{~cm}$ lange Dünndarmnippelventil leckte bereits bei $10 \mathrm{~cm} \mathrm{H}_{2} \mathrm{O}$, während die 3,4 und $5 \mathrm{~cm}$ langen Versionen maximalen Druckwerten von 73,100 und $125 \mathrm{~cm} \mathrm{H}_{2} \mathrm{O}$ widerstanden. 2. Die maximalen Intrareservoirdrucke für $3,4,5$ und $6 \mathrm{~cm}$ Durchzug lagen bei 74, 137, 80 und $118 \mathrm{~cm} \mathrm{H}_{2} \mathrm{O}$. Für die ileale Einbettung wurde ein Maximalwert von $94 \mathrm{~cm}$ $\mathrm{H}_{2} \mathrm{O}$ registriert, der Leckpunktdruck für das plikierte Ileum lag bei $40 \mathrm{~cm} \mathrm{H}_{2} \mathrm{O}$, für das eingeengte Ileum bei $25 \mathrm{~cm}$ $\mathrm{H}_{2} \mathrm{O}$. Für die neue Kontinenzventiltechnik, den KolonSerosa-Tunnel, lagen die Leckpunktdrucke für den 3, 5, 7 und $9 \mathrm{~cm}$ langen Kolon-Serosa-Tunnel bei 10, 75, 80 und $86 \mathrm{~cm} \mathrm{H}_{2} \mathrm{O}$. 


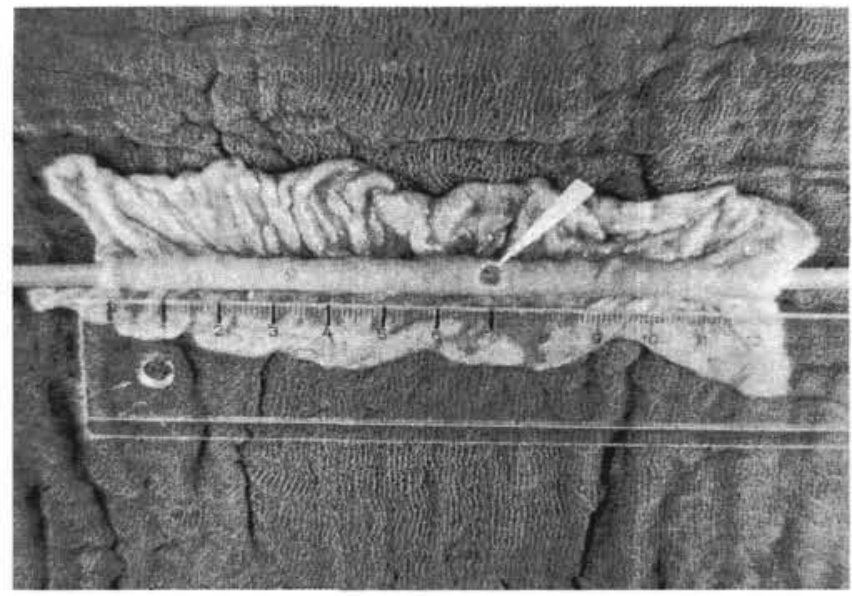

Abb. 1 Kolon-Serosa-Tunnel: Fensterung zum Reservoir bei $7 \mathrm{~cm}$ (Pfeil).

Die hydrodynamischen Ergebnisse des Invitro-Modells lassen sich direkt mit den aus der Literatur bekannten tierexperimentellen sowie klinischen Reservoirdrucken vergleichen. Diese sehr enge Korrelation bestätigt, daß mit dem Modell urodynamische Ergebnisse aller tierexperimentellen Studien sowie Patientenserien für die jeweilige Ventilart zu reproduzieren waren. Die ständige Verfügbarkeit von Schweinedarm, die einfache und unkomplizierte und noch dazu in sich völlig unproblematische Handhabung von Schweinedarm erlaubt eine preiswerte und zugleich der individuellen Kreativität nach allen Seiten hin offene Verwendung dieses In-vitro-Modells.

\section{Uro-Onkologie}

S. Madersbacher, Wien: Funktionelle Analyse von TPS. Koautoren der Arbeit waren: G. Steiner, G. Kramer, A. Schöllhammer, G. Hartmann, M. Marberger

Unkontrollierte Zellproliferationen sind ein charakteristisches Merkmal malignen Wachstums, wodurch sich die intensiven Forschungsaktivitäten hinsichtlich der Identifikation von Markern für abnormes Zellwachstum erklären. Zunehmendes Interesse wächst für solche Marker, die spezifisch für epitheliale Zellproliferationen (EC) sind, da die Tumorzelle ihre spezifischen Aufgaben verliert und infolgedessen aufhört, spezifische Tumorantigene zu sezernieren.

Eine der vielversprechendsten Marker dieses Typus ist TPS (spezifisches Epitop M3 des Tissue Polypeptide Antigen), ein Mitglied der heterogenen Gruppe von Molekülen, die in erster Linie durch Epithelzellen exprimiert werden.

Die vorgestellte Studie beschäftigte sich vorangig mit den Fragen: 1. Inwieweit ist die TPS-Produktion tatsächlich spezifisch für Epithelzellen? 2. Besteht eine Korrelation des epithelzellsezernierten TPS in-vitro mit DNA-Synthese, Zellüberleben und zunehmender Zellzahl? 3. TPS-Sekretion geht immer mit Zellnekrose einher und ist ein Produkt überlebender, proliferierender Zellen? 4.
Produzieren differenzierte PSA-negative Prostataepithelzellen signifikante Mengen an TPS in-vivo und in-vitro? 5. Läßt sich eine signifikante Korrelation zwischen SerumTPS und PSA bei Prostatakarzinom-Patienten feststellen?

Anhand eines direkten Vergleiches der meßbaren TPS-Mengen im Überstand der Kultur mit in die DNA inkorporiertem, radioaktivem Thymidin sowie mit der Reduktion von Tetrazoliumsalz konnte gezeigt werden, daß entgegen der üblichen Meinung, die TPS-Sekretion nicht von der Zellnekrose abhängt. Die Ergebnisse wurden weiter bestätigt durch die Tatsache, daß die Bestrahlung der Epithelzellen die TPS-Sekretion drastisch reduzierte. Unter Berücksichtigung der Tatsache, daß in Zellkulturen sowohl lebensfähige als auch nicht-lebensfähige Zellen in vergleichbaren Mengen zu finden sind, kann die TPS-Ausschüttung nicht von der Zellzahl abhängen. Demzufolge läßt sich, aufgrund der von Madersbacher et al. erhobenen Befunde ein direktes Abhängigkeitsverhältnis zwischen TPS-Sekretion und der Zellmitoserate feststellen. Die In-vitro-Assays dieser Gruppe, bei denen die meßbaren TPS-Mengen exklusiv in den Überständen der Zellkulturen zu finden und ausschließlich epithelialen Ursprungs waren, waren konsistent mit den Ergebnissen, die mittels Immundoppelfärbung unter Verwendung von Antizytokeratin erhoben worden sind.

Zur Überprüfung der klinischen Relevanz der In-vitro-Experimente wurde erstmals die Signifikanz von Serum-TPS als ein Tumorproliferationsmarker bei Prostatakarzinom-Patienten (PC) bestimmt. Beim lokalisierten PC waren die TPS-Spiegel vergleichbar zu denen von Patienten mit benigner Prostatahyperplasie und infolgedessen weniger verläßlich als das Serum-PSA. Patienten mit metastasiertem Prostatakarzinom zeigten einen signifikanten Anstieg der TPS-Konzentrationen, die nicht nur eng mit den erhobenen PSA-Spiegeln korrelierten, sondern in 2 von 25 Patienten eine signifikante TPS-Erhöhung zeigten bei normalem PSA-Wert.

Neben dem rein klinischen Nutzen scheint die TPS-Bestimmung von großem Interesse zu sein für die Basis- sowohl als auch die pharmazeutische Forschung, da TPS-positive Prostataepithelzellinien ihre Fähigkeit verlieren signifikante Mengen von PSA unter In-vitro-Bedingungen $\mathrm{zu}$ produzieren, was für TPS nicht zutrifft. Schließlich muß diskutiert werden, inwieweit die TPS-Bestimmung eine noch bedeutendere Rolle in der Diagnose des Nieren- und Urothelkarzinoms spielen kann. Vorläufige Ergebnisse deuten auf vielversprechende Ergebnisse hin.

E. C. De Boer, Amsterdam: Signifikanz des Urinalbuminanstiegs nach BCG-Behandlung des oberflächlichen Blasenkarzinoms. Veränderungen der HLA-ABCund HLA-DR-Antigen-Expression urothelialer Zellen nach BCG-Behandlung. Koautoren dieser Arbeit waren: D. H. J. Schamhart, W. H. De Muinck Keizer, Th. M. De Reijke, K. H. Kurth

Unbeachtet des guten Ansprechens intravesikaler BCG-Instillationsbehandlung beim oberflächlichen Blasenkarzinom sind akurate, prognostische Indika- 
toren mit ausreichender Spezifität zur frühen Erkennung von Therapie-Nonrespondern dringend notwendig, um alternative Behandlungsformen vor einer weiteren Tumorprogression einleiten zu können. Aus diesem Grund wurden alle Patienten während der ersten sechs Wochen der BCG-Instillationsbehandlung immunologisch überwacht, wobei verschiedene Parameter bestimmt wurden: Zytokine, Urinalbumin, HLA-ABC- und HLA-DR-Expression von Urothelzellen.

Sowohl Albumin als auch die Zytokine IL1 $\beta$, IL2, IL6 und TNF $\alpha$ wurden in festgelegten Schritten bestimmt: vor der Instillationsbehandlung und von 2 bis 24 Stunden nach jeder der 6wöchentlichen BCG-Instillationen.

Der Anstieg des Urinalbumins schien eng mit dem Zytokinanstieg (IL1 $\beta$, IL2, IL6 und TNF $\alpha$ ) im Urin nach BCG-Behandlung zu korrelieren. Während der ersten der 6wöchentlichen BCG-Instillationen zeigte sich, daß sowohl die Urinzytokine als auch das Albumin durch a) wiederholte Installationsbehandlungen anstiegen und b) insbesondere während der ersten 2-6 Stunden nach der Instillationsbehandlung eine deutliche Konzentrationszunahme zu verzeichnen war. Bei Patienten, die keinen Zytokinanstieg zeigten, ließ sich auch keine Albuminzunahme nachweisen (Abb. 1).

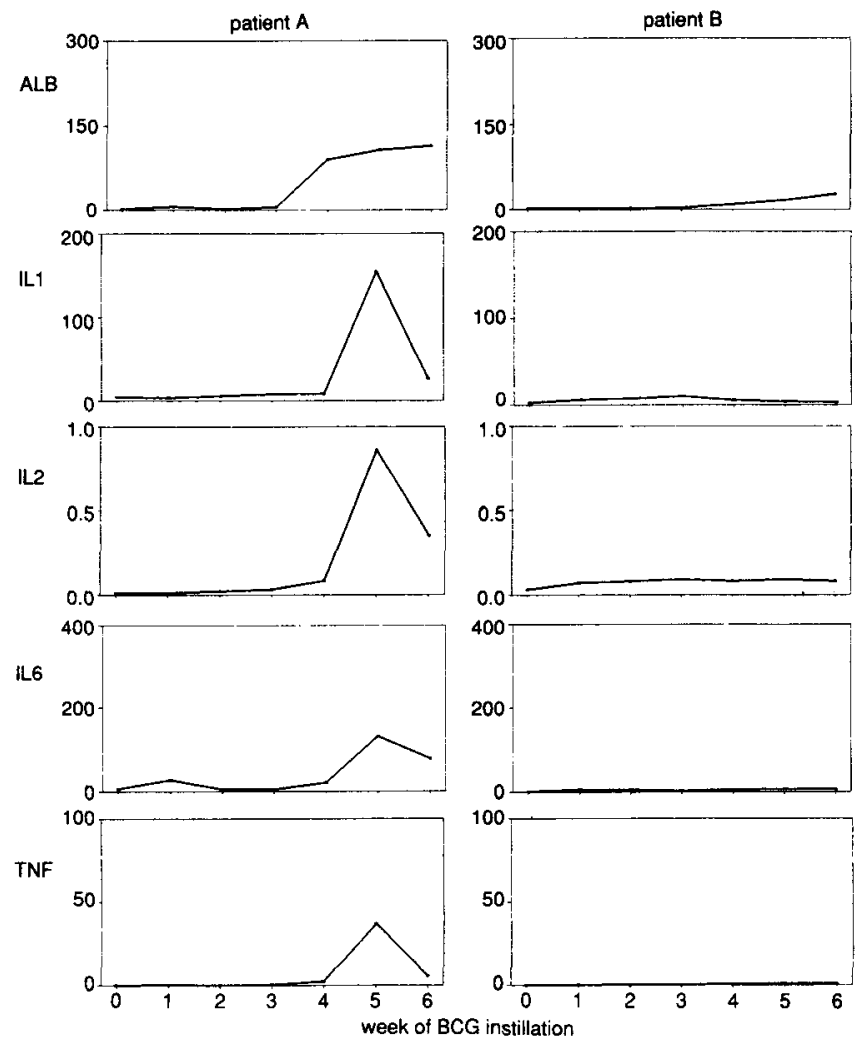

Abb. 1 Albumin und Zytokine im Urin während der 6wöchigen BCGBehandlung bei 2 representativen Patienten. Albumin (ALB) exprimiert als $\mu \mathrm{g} / \mu$ mol creat; IL 1 $\beta$, IL6 und TNF $\alpha$ als pg/ $\mu$ mol creat; IL.2 als $\mathrm{U} / \mu \mathrm{mol}$ creat.
Die Expression von humanem LeukozytenAntigen (HLA)-ABC- und HLA-DR-Molekülen auf der Zelloberfläche ist notwendig, um Antigene gegen T-Suppressor/Zytotoxische (CD8+)-Zellen und T-Helfer/Induktions(CD4+)-Zellen für die Induktion einer immunologischen Reaktion zu erhalten. Immunhistologische Untersuchungen an Gewebebiopsaten anderer Untersucher hatten gezeigt, daß die Induktion von HLA-DR-Molekülen am Urothel nach intravesikaler BCG-Instillation stattfindet. Die Amsterdamer Gruppe untersuchte die HLA-ABC- und HLA-DR-Expression von Urothelzellen der Harnblase anhand von wash out(BWO)-Proben. Mittels einer direkten Dreischritt-Immunperoxidase-Methode wurden die Veränderungen der 6 wöchigen Therapie jeweils vor der 1 ., $3 ., 5$. und 6 . Instillation bestimmt.

Bei allen bisher untersuchten Patienten $(n=8)$ war die HLA-ABC- und HLA-DR-Expression vor der ersten Behandlung gering oder nicht nachweisbar. Während der Therapie zeigte sich ein deutlicher Anstieg für die HLA-ABC- und HLA-DR-Expression bei 5 Patienten, wohingegen die anderen 3 Patienten keine Veränderung der HLA-Expression zeigten. Bei letztgenannten Patienten wurde ebenso keine Urinzytokininduktion beobachtet.

Zusammenfassend stellt Frau De Boer fest, daß die BCG-Behandlung rein empirischer Art ist und Grundlagenwissen über das optimale Behandlungsschema zur Immunstimulation und Antitumoraktivität spärlich ist. Ein möglicher Weg zur Optimierung der Therapie könnte die individuelle Anpassung des Therapieschemas sein, beispielsweise wöchentliche BCG-Instillationen bis eine immunologische Antwort gesichert ist, z. B. über den Anstieg des Urinalbumins. Des weiteren könnte die Effektivitätsbeurteilung der BCG-Behandlung über die Zunahme der HLA-Expression von urothelialen Tumorzellen in BWO dienen, die ebenfalls mit der Urinzytokininduktion stark assoziiert ist und, wie zuvor aufgezeigt, problemlos bestimmt werden kann. Eine fragliche Korrelation zwischen HLA-Expression von Urothelzellen in BWO und Biopsien im Vergleich mit dem klinischen Ansprechen sind derzeit Gegenstand einer weitergehenden Untersuchung.

\section{G. H. Mickisch, Rotterdam: Krebsvielfachresistenz - Experimentelle Fortschritte und klinische Konzepte}

In den letzten Jahren wurden - u. a. durch Erschließen der Molekularbiologie - große Fortschritte in der Resistenzforschung erzielt. So konnte das P-Glykoprotein, eine Membranpumpe, die vom MDR1-Gen kodiert wird, als einer der wesentlichen Resistenzmechanismen in vielen menschlichen Tumoren nachgewiesen werden. Im Bereich der urologischen Onkologie scheint es vor allem beim Nierenzellkarzinom und beim rezidivierten, vorbehandelten Blasenkarzinom von funktioneller Bedeutung für das Auftreten von Chemoresistenz zu sein. Arbeiten an einem neuartigen Tiermodell, der sogenannten MDR1transgenen Maus, die das menschliche Resistenzgen funktionell exprimiert, konnten zeigen, daß Überwindung von Resistenz durch Hemmung von P-Glykoprotein möglich und für einige Tumorarten wahrscheinlich auch sinnvoll 
ist. Dieser Vorgang wird Chemosensitivierung genannt. Auf der Basis neuentwickelter, effektiver und nebenwirkungsarmer Chemosensitivierer wird derzeit eine Reihe klinischer Studien an soliden und hämatologischen Tumoren durchgeführt. Da die bislang publizierten Daten positiv klingen, ist kürzlich weltweit eine Reihe verschiedener Studien mit diesem neuen Wirkprinzip am Nierenzellkarzinom begonnen worden (Tab. 1). Ein experimentell interessanter Ansatz, der nun zur klinischen Prüfung ansteht, sind die Studien der Rotterdamer Universitätsurologie. Hier wird Dexverapamil, der derzeit nebenwirkungsärmste Chemosensitivierer der 2. Generation mit Vinblastin, dem einzigen Chemotherapeutikum mit dokumentierten Ansprechraten beim Nierenzellkarzinom kombiniert. Ferner wird die Effizienz der Kombination durch u.a. Niederregulieren von Glukokortikoidrezeptoren der Zellwand mit Dexamethason verbessert.

Tab. 1 Klinische Studien zur Überwindung von Multidrug Resistance beim Nierenzellkarzinom.

\begin{tabular}{lll}
\hline Chemotherapeutikum & Chemosensitivierer & Applikation* \\
\hline Vinblastin & Cyclosporin A & intravenös \\
Vinblastin & $\mathrm{D}(+/+)$ Niguldipin & oral \\
Vinblastin & $\mathrm{D}(+/+)$ Niguldipin & intravenös \\
Vinblastin & Dexverapamil & oral \\
Doxorubicin & Dexverapamil & oral \\
Vinblastin** & Dexverapamil und & oral \\
& Dexamethason & \\
Vinblastin** & Dexverapamil und & intravenös \\
& Dexamethason & \\
\hline
\end{tabular}

* des Chemosensitivierers ** Rotterdamer Urologie

L. Hertle, Münster: Nutzen, Nebenwirkungen und Kosten der Immuntherapie mit Interferon und Interleukin beim fortgeschrittenen, metastasierten Nierenzellkarzinom. Koautor dieser Arbeit war: P. Faber

Hertle berichtete über 34 Patienten mit metastasiertem Nierenzellkarzinom, die von Mai 1990 bis November 1992 in der Münster Universitätsklinik behandelt worden waren. 21 Patienten hatten eine Kombinationstherapie aus Interferon- $\alpha 2 b$ und Interleukin- 2 erhalten. Die ersten 5 dieser Patienten erhielten relativ hohe Dosierungen und eine kontinuierliche intravenöse Applikation von Interleukin-2. Die Hospitalisationszeit betrug unter dieser Therapie ca. $60 \%$ der Überlebenszeit. Alle Patienten zeigten schwere Nebenwirkungen wie Fieber, Abgeschlagenheit und Schlaflosigkeit. Bei einem Patienten erfolgte ein Therapieabbruch wegen beginnendem Leber- und Nierenversagen.

Weitere 15 Patienten wurden mit einer Niedrigdosistherapie zeitweise stationär, jedoch überwiegend ambulant behandelt. Die Applikation von Interferon und Interleukin erfolgte subkutan, die Hospitalisationszeit betrug im Schnitt nicht mehr als 12,5\% der Überlebenszeit. Trotz niedrigerer Dosierung klagten 12 von 15 Patienten über ein ständiges ,Grippegefühl'. Nach 2

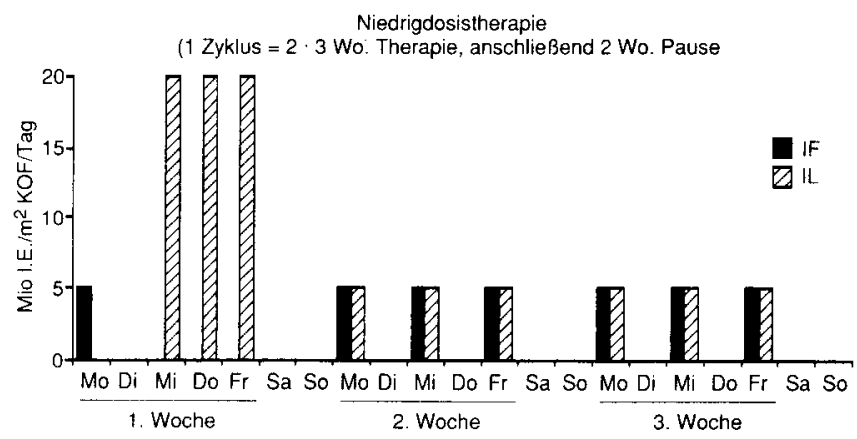

Abb. 1

Zyklen als Induktionstherapie (Abb. 1) wurde bei stabilem Verlauf oder bei Remission eine Erhaltungstherapie angeschlossen. Die Kosten für einen sechswöchigen Therapiezyklus betrugen ca. DM 15000 , die der bis zum Progre $\beta$ durchgeführten Erhaltungstherapie ca. DM 2000 pro Monat.

Profitiert haben von der Kombinationstherapie bisher 3 Patienten (2 komplette und 1 partielle Remission). Abgebrochen werden mußte die Therapie bei 9 Patienten mit Progression und bei 5 Patienten wegen erheblicher Nebenwirkungen. Hierbei waren die Patienten nicht bereit, das mit der Therapie einhergehende ständige Grippegefühl zu ertragen. Faßt man die letzten beiden Gruppen zusammen, so haben 14 der bisher behandelten Patienten keinen Nutzen aus der Therapie ziehen können. 4 weitere Patienten stehen zur Zeit mit stable disease unter Therapie. Hier muß der weitere Verlauf abgewartet werden.

In der Literatur werden Ansprechraten bis $35 \%$, komplette Remissionen bis $15 \%$ und eine Stabilisierungs- bzw. Remissionsdauer bis 6 Monate angegeben.

Zusammenfassend ist festzustellen, daß bei starker Beeinträchtigung der Lebensqualität, fehlender Aussicht auf Heilung und hohen Therapiekosten eine Kombinationstherapie weder eine Standardtherapie noch eine „Plazebotherapie“ (besser diese als gar nichts) darstellt und Immunmodulatoren nur im Rahmen kontrollierter Studien angewendet werden sollten.

T. Kato, Akita, Japan: Zielgerichtete Krebschemotherapie mit Mikrokapsel-Chemoembolisation. Koautoren der Arbeit waren: K. Sato, M. Moriyama

Die transkatherale arterielle Infusion von mikroinkapsulierten Chemotherapeutika war als Prototyp zur zielgerichteten Karzinomchemotherapie entwickelt worden. Der erzielbare therapeutische Effekt resultiert aus der Mikroinfarzierung des okkludierten Gebietes sowie der verlängerten Substanzwirkung am Zielorgan, was diesem Therapieverfahren den Begriff der ,Chemoembolisation'verliehen hat.

Sowohl tierexperimentelle als auch klinische Studien haben einen verlängerten Antitumoreffekt 
mit Reduktion der systemischen Toxizität gezeigt im Vergleich zu traditionellen regionalen Chemotherapieverfahren.

Kato berichtete über die Erfahrung mit 759 Patienten, die chemoembolisiert worden waren. Die Autoren kamen zu dem Schluß, daß eine MikrokapselChemoembolisation für verschiedene Tumorläsionen verwendet werden kann, soweit eine selektive arterielle $\mathrm{Ka}$ theterisierung möglich ist, die akzeptable Ergebnisse hinsichtlich des Antitumoreffekts sowie den Nebenwirkungen bzw. Komplikationen zuläßt. Bei invasiven Tumoren wird durch die vorherige Chemoembolisation der chirurgische Eingriff häufig erleichtert und eine deutliche Reduktion der Blutungsneigung bzw. der Schmerzsensation beim Patienten erreicht. Zusätzlich kann die postoperative Überlebensrate bei präoperativer, adjuvanter Chemoembolisation verbessert werden.

Die Autoren kommen zu dem Schluß, daß die Ergebnisse, die Verfügbarkeit und Effizienz der Chemoembolisation als zielgerichtete Karzinomchemotherapie belegen und die Rechtfertigung für weitere Studien untermauern.

\section{Neurourologie und Urodynamik}

L. Baskin, Philadelphia: Der Einfluß physikalischer Kräfte an der glatten Blasenmuskulatur und urothelialen Zellmatrixsynthese. Koautoren der Arbeit waren: $P$. Howard, J. Duckett, H. Snyder, E. Macarak

Alterationen der Blasenphysiologie können durch Obstruktion (erhöhter Druck) und/oder neurologische Pathologien bedingt sein. Derartige Veränderungen resultieren in einer Bindegewebezunahme (Kollagen Typ I und III sowie Fibronectin) der Blasenmuskulatur mit konsekutiver Abnahme der Blasen-Compliance. Um den Einfluß von Druckänderungen (physikalische Kräfte) an der Blase unter Ausschluß der nervalen Innervation untersuchen zu können, wurde ein zelluläres Modellsystem entwickelt, dem die Isolation der beiden primären Zelltypen der Blase glatter Muskel und urotheliale Zellen zugrunde liegt.

Die extrazelluläre Matrix-Protein-Biosynthese dieser beiden Zellinien im In-vitro-Modell wurde durch Markierung des Proteins mit C14 radioaktivmarkiertem Prolin und anschließender Gelelektrophorese charakterisiert. Die Untersuchungen zeigten, daß sowohl die Blasenmuskulatur als auch die Urothelzellen Fibronectin und interstitielles Kollagen, Typ I und II, synthetisieren. Da Blasenzellen in einem aktiven physikalischen Umfeld leben, wurde versucht, diese Situation auf

\begin{tabular}{llll}
\hline Zelltyp & Kollagen Typ I & Typ III & Fibronectin \\
\hline glatter Muskel & $\uparrow^{*}$ & $\downarrow^{*}$ & $\uparrow *$ \\
Urothel & $\uparrow *$ & $\leftrightarrow$ & $\uparrow *$ \\
\hline
\end{tabular}

$* p<0,005$ zellulärer Ebene zu simulieren. Unter Verwendung eines eigens entwickelten Systems wurde eine exakt vorgegebene, standardisierte und reproduzierbare mechanische Deformation (physikalische Kraft) auf beide Zelltypen gegeben. Mittels ELISA wurde die Kollagen-Typ-I- und -III sowie Fibronectinsynthese, sowohl der glatten Blasenmuskulatur als auch der Urothelzellen, unter mechanischer Belastung (Deformation) ( $\mathrm{Zug}=4,9 \%$ ) untersucht. Die Ergebnisse wurden mit denen von Kontrollzellen verglichen, die bis auf die physikalische Deformation den gleichen experimentellen Voraussetzungen ausgesetzt waren.

Zusammenfassend kommt die Gruppe zu dem Schluß, daß durch die Entwicklung eines zellulären In-vitro-Blasenmodells die Möglichkeit gegeben ist, die extrazelluläre Matrix-Expression der Blase zu untersuchen. Zusätzlich konnte gezeigt werden, daß die extrazelluläre Matrix-Synthese durch physikalische Kräfte moduliert wird (=noncompliant Blase), sowohl bei der glatten Blasenmuskulatur als auch bei der Urothelzelle. Die vorgestellte Untersuchung unterstreicht die Hypothese, daß die Blasenfunktion allein durch unterschiedliche Druckphänomene ohne weitere Einflußnahme neurologischer Aspekte verändert werden kann, was dem Verständnis der Pathophysiologie einer obstruierten Blase dienlich ist.

C. E. Constantinou, Standorf, USA: Experimentelle Modelle zur Untersuchung der BPH bei der Ratte

Die Einflußnahme von Dihydrotestosteronpropionat (DHT) und Estradiol (E) auf das Prostatawachstum und den volumenevozierten Miktionsreflex (VEMR) wurden an 22 Sprague-Dawley-Ratten über einen 28tägigen Zeitraum untersucht. Die Ratten wurden in 3 gleiche Gruppen unterteilt: 1. Kontrollgruppe, 2. DHT und 3. DHT + E, wobei jede Gruppe täglich 0,1 ml Sesamöl vermischt mit $1,25 \mathrm{mg}$ DHT für Gruppe 2 und $1,25 \mathrm{mg}$ DHT +0,125 mg E für Gruppe 3 zusätzlich erhielt. Die physiologischen Messungen des VEMR wurden in 3- bis 4tägigen Abständen durchgeführt, wobei $10 \mathrm{mg} / \mathrm{kg}$ KG Furosemid $+5 \mathrm{ml}$ Kochsalz subkutan vor Beginn eines jeden Experiments den Ratten injiziert wurden.

Die Ergebnisse wurden mittels ANOVA analysiert und die eigenen Ergebnisse als Mittelwerte \pm Standardabweichung wiedergegeben.

Das Prostatagewicht sah wie folgt aus: 1. Kontrollgruppe: $0,89 \pm 0,06 ; 2$. DHT-Gruppe: 1,26 $\pm 0,10 ; 3$. DHT +E-Gruppe: $1,24 \pm 0,07 \mathrm{gm}$. Prozentual ausgedrückt bedeutet das eine 42 bzw. $40 \%$ ige signifikante $(p<0,01)$ Zunahme des Prostatagewichtes für die DHTund DHT + E-Gruppe im Vergleich zu der Kontrollpopulation. Kein signifikanter Unterschied bestand zwischen Gruppe 2 (DHT) und 3 (DHT+E). Die Analyse der Miktionsparameter zeigte, daß das mittlere Miktionsvolumen, sowohl in der DHT als auch der DHT $+\mathrm{E}$ behandelten Gruppe, deutlich reduziert waren. Die Gruppe, die eine DHT-Monotherapie erhalten hatte, zeigte deutlich geringere Miktionsvolumina ( $p<0,05)$ nach 14 Tagen. Im Zeitraum von 14 bis 24 Hormonbehandlungstagen zeigten beide Gruppen, sowohl die DHT- als auch die DHT +EGruppe, signifikant reduzierte mittlere Miktionsvolumina 
( $p<0,01$ ). Die niedrigsten Miktionsvolumina wurden nach 17 Tagen für die DHT-Gruppe gemessen, wobei sich im Vergleich zur Kontrollgruppe das mittlere Miktionsvolumen von $3,04 \pm 0,27 \mathrm{ml}$ auf $1,68 \pm 0,05 \mathrm{ml}(\mathrm{p}<0,01)$ reduzierte; analog der Ergebnisse der DHT +E-Gruppe: 1,86 $\pm 0,31 \mathrm{ml}(\mathrm{p}<0,01)$. Kein signifikanter Unterschied ergab sich hinsichtlich der Miktionsfrequenz zwischen der Kontrollgruppe und den hormonbehandelten Tieren.

Die Ergebnisse zeigen eine deutliche und signifikante Beeinträchtigung der Miktionscharakteristik (VEMR). Der Autor kommt zu dem Schluß, daß das vorgestellte Rattenmodell für häufige Untersuchungen der BPH unter Androgen-/Östrogen-Einfluß geeignet ist. Durch Einbringen weiterer Miktionsparameter zu den vorhandenen, zugänglichen morphologischen Veränderungen scheint dieses Modell ideal für die Untersuchung der pharmakologischen Wirkungsweise unterschiedlicher Substanzen zur Therapie der BPH.

\section{- H. Nawrath, Mainz: Elektrophysiologische Methoden zur Untersuchung zellulärer Erregungsabläufe}

Die Exzitations-Kontraktionskopplung in Herz-, Skelett- und glatter Muskulatur kann durch eine Reihe von Rezeptorsystemen, die an der Außenseite der Zellmembran lokalisiert sind, moduliert werden. Endogene Neurotransmitter, die von den synaptischen Terminalen freigesetzt werden, binden sich an diese Rezeptoren und lösen eine Kaskade biochemischer Vorgänge aus, die Zellfunktionen in vielfältiger Weise ändern. Bei der Signalübertragung kann eine Reihe 'intracellular messengers', wie z.B. zyklische Nukleotide, Inositol-Triphosphat und $\mathrm{Ca}^{++}$, beteiligt sein. Diese biochemischen Ereignisse führen - neben anderen Veränderungen - zu funktionellen Alterationen der membrandurchdringenden Ionenkanäle der Lipiddoppelschicht.

Ein Ziel elektrophysiologischer Untersuchungen besteht nun darin, die Mechanismen der Rezeptoraktivierung und der kontraktilen Zellfunktionen unter normalen und pathophysiologischen Bedingungen zu untersuchen. Ein großer Schritt vorwärts wurde hier durch die Einführung der 'patch clamp'-Technik (Membranfleckklemme) erreicht, die es ermöglicht, neben den Ionenströmen erregbarer Zellen auch die elektrischen Aktivitätsänderungen einzelner Kanäle zu untersuchen.

Ein Beispiel dafür ist die Erklärung der Wirkungsweise von 5-Hydroxytryptamin (5-HT) auf die menschliche Herzmuskulatur durch die Verwendung der patch clamp-Technik. 5-HT führt zu einem positiven inotropen Effekt, der mit einem Anstieg des $\mathrm{Ca}^{++}$-Stromes $\left(\mathrm{I}_{\mathrm{ca}}\right)$ in den rechten Vorhof verbunden ist. Patch clamp-Untersuchungen weisen darauf hin, daß der 5-HT-induzierte Anstieg des makroskopischen $\mathrm{I}_{\mathrm{ca}}$ im menschlichen Vorhof eine erhöhte Aktivierbarkeit von $\mathrm{Ca}^{++}$-Kanälen hinsichtlich der Öffnung nach Depolarisation widerspiegelt. Der Schritt vom nicht-aktivierbaren zum aktivierbaren $\mathrm{Zu}$ stand der $\mathrm{Ca}^{++}$-Kanäle, wodurch sich deren Öffnungsverhalten ändert, kann auch als eine cAMP-abhängige Phosphorylierung des Kanalproteins - als eine Reaktion auf 5HT - gedeutet werden.
M. Hohenfellner, Wuppertal: Reinnervation der Blase mit autologen somatomotorischen Transplantaten. Koautoren dieser Untersuchung waren: $J$. W. Thüroff, $R$. A. Schmidt, E. A. Tanagho

Im Gegensatz zu einer oberen motorischen Rückenmarksverletzung mit der Option der sakralen Vorderwurzelstimulation zur Wiederherstellung einer willkürlich steuerbaren Miktion, sind bei motorischer Denervation der Blase mit konsekutiver Detrusorhypo- bzw. Areflexie, infolge einer infranukleären oder sakralen Läsion, die therapeutischen Möglichkeiten begrenzt. In einem chronischen Rattenmodell wurde die Reinnervation der denervierten Harnblase durch ein somatomotorisches Nerven- oder ein quergestreiftes Muskellappentransplantat untersucht.

Nach Denervierung des Detrusors durch unilaterale Exzision des linken Ganglion pelvicum wurde entweder der linke N. femoralis (Gruppe I) oder ein Segment des quergestreiften $M$. pectineus (Gruppe II) in die Blasenmuskulatur autotransplantiert. Vier Monate später erfolgte die komplette Blasendenervierung durch Exzision des rechten Ganglion pelvicum und die Nerven-/Muskeltransplantate wurden elektrostimuliert unter simultaner Blasendruckmessung. Die direkte Injektion von Meerrettichperoxidase (HRP) in den Detrusor bzw. WGA-apo HRP-Gold in die Transplantate, die beide über einen schnellen retrograden Transport zu den Nervenzellkörpern transportiert werden, erlaubte die neuroanatomische Verbindung zwischen Detrusor und Transplantant zu beurteilen.

Sowohl in Gruppe I $(8 / 10)$ als auch in Gruppe II (7/10) konnte durch Elektrostimulation des Nerven- bzw. Muskellappentransplantats eine Detrusorkontraktion ausgelöst werden, die in Gruppe I (N. femoralis) durch Atropin $(0,5 \mathrm{mg} / \mathrm{kg} \mathrm{KG} \mathrm{i.v.)} \mathrm{unterdrückt} \mathrm{wur-}$ de, in Gruppe II (Muskeltransplantat) dagegen persistierte. In Gruppe I wurde HRP aus dem Detrusor in die motorischen vorderen Zellen des $\mathrm{N}$. femoralis transportiert, dieses Phänomen wurde allerdings nicht in Gruppe II (Muskeltransplantat) beobachtet.

Die vorgestellten Untersuchungen zeigen, daß eine Reinnervation glattmuskulärer Organe mit somatomotorischen Transplantaten prinzipiell möglich ist. Die neuroanatomischen Studien belegen eine neuronale Verbindung zwischen Detrusor und Nerventransplantat, die eine der originalen, autonomen Innervation ähnliche Reinnervation vermuten läßt. Beim Muskeltransplantat wurde die Detrusorkontraktion vermutlich durch einen direkten Stromfluß zwischen Transplantat und Zielorgan verursacht.

$K$.-P. Jünemann, Mannheim: Koexistenz von NO-Synthase und VIP in Nervenendigungen des Corpus cavernosum. Koautoren der Arbeit waren: H. Ehmke, W. Kummer, P. Alken

Als primären, neuronalen Transmitter einer nicht-adrenergen, nicht-cholinergen (NANC) übermittelten, glattmuskulären Relaxation des erektilen Gewebes 
werden die Neuropeptide: vasoaktives intestinales Polypeptid (VIP) und Stickoxid (NO), vormals als EDRF bezeichnet, angesehen. NO-Synthase (NOS) konnte trotz mehrfacher Versuche bisher nicht in humanem Schwellkörpergewebe nachgewiesen werden. Vorangegangene Grundlagenarbeiten über Existenz und Wirkungsweise von VIP im Schwellkörpergewebe sowie das zunehmende Interesse an NO-Synthase waren ausschlaggebend für den Versuch, NOS im erektilen Gewebe zu identifizieren und zu lokalisieren und seine mögliche Koexistenz zu anderen Neuropeptiden zu bestimmen.

Im Rahmen peniler Prothesen- bzw. Revaskularisationschirurgie wurden von 6 impotenten Patienten offene Schwellkörperbiopsien entnommen und einer Immunhistochemie und NADPH-Diaphorase-Reaktion zur Identifikation von NOS unterzogen. Zur Koexistenzbestimmung weiterer neuraler Mediatoren in NOS-positiven Axonen wurde die NOS-Immunhistochemie mit Antiseren gegen VIP, Tyrozin-Hydroxylase und Substance P kombiniert.

Die Immunhistochemie sowie die NADPHDiaphorase-Reaktion zeigten eine deutliche NOS-Immunreaktivität in den die glatte Schwellkörpermuskulatur innervierenden Axonen sowie in Nervenfasern, die zu den korkenzieherartigen Arterien im Penis ziehen. Im Gegensatz zu der ausgeprägten axonalen Reaktivität zeigten die Endothelzellen des Corpus cavernosum eine nur schwache NOS-Färbung. Die Doppelfärbung ergab einen hohen Koexistenzgrad von NOS- und VIP-Immunreaktivität in den perivaskulären Axonen ( $>50 \%$ ) sowie den trabekulären Nervenfasern des Corpus cavernosum (>90\%), wohingegen nonadrenerge und Substance P-haltige Nervenfasern keine NOS-Immunreaktivität aufwiesen.

Die Immunfärbungen für NOS und VIP waren in allen Biopsien vergleichbar bis auf einen Patienten mit neurogener Impotenz nach radikaler Zystoprostatektomie aufgrund eines Blasentumors. In diesem Fall konnte weder eine NOS- noch eine VIP-positive Färbung der Nervenfasern im Corpus cavernosum nachgewiesen werden, was auf eine komplette kavernöse Nervenläsion hinweist. Ein zweiter Patient mit einem seit 18 Jahren insulinabhängigen Diabetes mellitus zeigte eine Reduktion der NOS-positiven Immunreaktivität um ca. $50 \%$ gegenüber den vier anderen Patienten.

Die Autoren kommen zu dem Schlu $\beta$, da $\beta$ der hohe Grad einer Koexistenz von NOS- und VIP-positiver Immunreaktivität in kavernösen Nervenfasern impliziert, daß NO nicht der alleinige Neurotransmitter der penilen Erektion sein kann. Die Ergebnisse lassen vermuten, daß NOS und VIP als Komediatoren bei der penilen Erektion fungieren.
PD Dr. med. K.-P. Jünemann

Urologische Klinik

Klinikum Mannheim

Universität Heidelberg

Theodor-Kutzer-Ufer 1

D-68135 Mannheim 